\title{
Pourquoi les optométristes devraient-ils commencer un blogue?
}

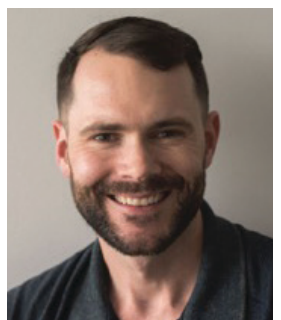

Cameron Martel

Cameron Martel est un spécialiste du marketing numérique chevronné qui gère des campagnes de SEO et de contenu depuis 2005. Il travaille actuellement avec des dizaines de cabinets de soins oculovisuels dans le cadre de son travail avec Marketing4ECPs. Il est daltonien, mais ne le lui rappelez pas, sinon il verra rouge! (ou du moins, c'est ce qu'il croit). Pour joindre Cameron :www.marketing4ecps.com ou cameron@4ecps.com.

$\mathrm{N}$ ous encourageons fortement les cabinets d'optométrie à créer un blogue sur les pratiques au sein de l'industrie. Il s'agit de l'un des moyens les plus efficaces de générer du trafic supplémentaire, d'accroître la visibilité de votre marque et d'attirer de nouveaux patients. C'est pourquoi nous en avons fait l'un des piliers de notre stratégie de SEO (search engine optimization, ou optimisation pour les moteurs de recherche en français).

De nos jours, les blogues sont moins en avant-plan, et ceux qui en rédigent s'y prennent souvent de la mauvaise manière. Bien des cabinets concentrent davantage leurs efforts sur les médias sociaux et ajoutent rarement, pour ne pas dire jamais, du nouveau contenu sur leur blogue. Voyons ensemble pourquoi il s'agit d'une occasion ratée pour eux, et d'une occasion en or pour vous.

POURQUOI LE BLOGUE EST-IL L'UN DES OUTILS DE RÉFÉRENCEMENT NATUREL LES PLUS PUISSANTS?

Google raffole du contenu de qualité. Google adore lorsque ses utilisateurs se perdent dans les dédales des résultats de recherche, enchainnant les blogues l'un après l'autre et absorbant ainsi de grandes quantités d'informations.

Pourquoi?

Parce que cela signifie que ses utilisateurs trouvent utiles les résultats que Google leur propose, et que si c'est réellement le cas, ces derniers reviendront encore et encore. Compte tenu du fait que Google détient plus de $90 \%$ des parts du marché des moteurs de recherche, nous avons des raisons de croire que sa logique est implacable.

Il s'agit également de l'un des moyens les moins coûteux de cibler des clients potentiels. Par ailleurs, si vous créez le blogue vous-même, il vous en coûtera uniquement un peu de votre temps. Lorsqu'il est géré correctement, un blogue peut réellement transformer les activités d'une entreprise. Sans l'ombre d'un doute!

\section{LES POSSIBILITÉS OFFERTES PAR LE BLOGUE}

Les gens qui sous-estiment les blogues oublient deux points importants : en premier lieu, les utilisateurs veulent accéder à cette information et cherchent activement à l'obtenir; deuxièmement, Google souhaite que ces renseignements proviennent d'experts en la matière comme vous. En fait, Google a apporté un changement important à son algorithme en août 2018 afin de privilégier le contenu provenant de professionnels de la santé dans les résultats de recherche associés à des mots-clés relatifs à la médecine. Autrement dit, les optométristes disposent d'un avantage important en ce qui a trait aux mots-clés concernant la santé oculaire.

Les blogues comportent quatre principaux avantages d'un point de vue opérationnel :

\section{Ils vous permettent de renseigner et de sensibiliser vos futurs patients et clients.}

Combien de fois avez-vous dû justifier le prix d'un examen de la vue ou d'une monture? La réalité est que la plupart des gens sont tout simplement inconscients des choses qu'ils ignorent. Par l'entremise de votre blogue, vous pouvez informer vos lecteurs au sujet de la santé oculovisuelle, de la correction de la vue et des articles de lunetterie et tout ce qui s'en rapporte. 
Un patient renseigné est plus susceptible de reconnaître la valeur de ce que vous lui proposez et beaucoup moins enclin à faire des achats en se fondant uniquement sur le prix.

\section{Ils vous permettent de démontrer votre expertise et votre leadership.}

À l'ère des fausses nouvelles et de la désinformation, les gens cherchent à obtenir des renseignements auprès d'experts auxquels ils peuvent véritablement faire confiance. Si vous parvenez à les informer sur un sujet qui leur tient à cœur, vous gagnerez leur confiance.

Le contenu que vous créez pour votre blogue peut facilement être partagé sur vos réseaux sociaux, y compris LinkedIn. En répondant aux questions de vos lecteurs, vous gagnerez leur confiance et leur appui. Voilà quelque chose de puissant que vous ne pouvez accomplir par l'entremise de la publicité traditionnelle.

\section{Ils vous permettent d'améliorer votre référencement dans Google et le trafic qu'il génère.}

Il y a énormément de trafic sur le Web. La phrase « comment fonctionnent les lunettes pour personnes daltoniennes » fait l'objet d'une recherche plus de 1300 fois par mois aux États-Unis et 150 fois par mois au Canada. Ajoutez à cela les différentes variantes (telles que « les lunettes pour personnes daltoniennes sont-elles efficaces ») et les chiffres augmentent de manière significative.

\section{Aperçu par mot-clé : « comment fonctionnent les lunet tes pour personnes daltoniennes " \\ Base de données : 監 États-Unis $\vee \mid$ Appareil : $\square$ ordinateur de bureau Date : 8 janvier $2020 \mid$ Devise : \$ US}

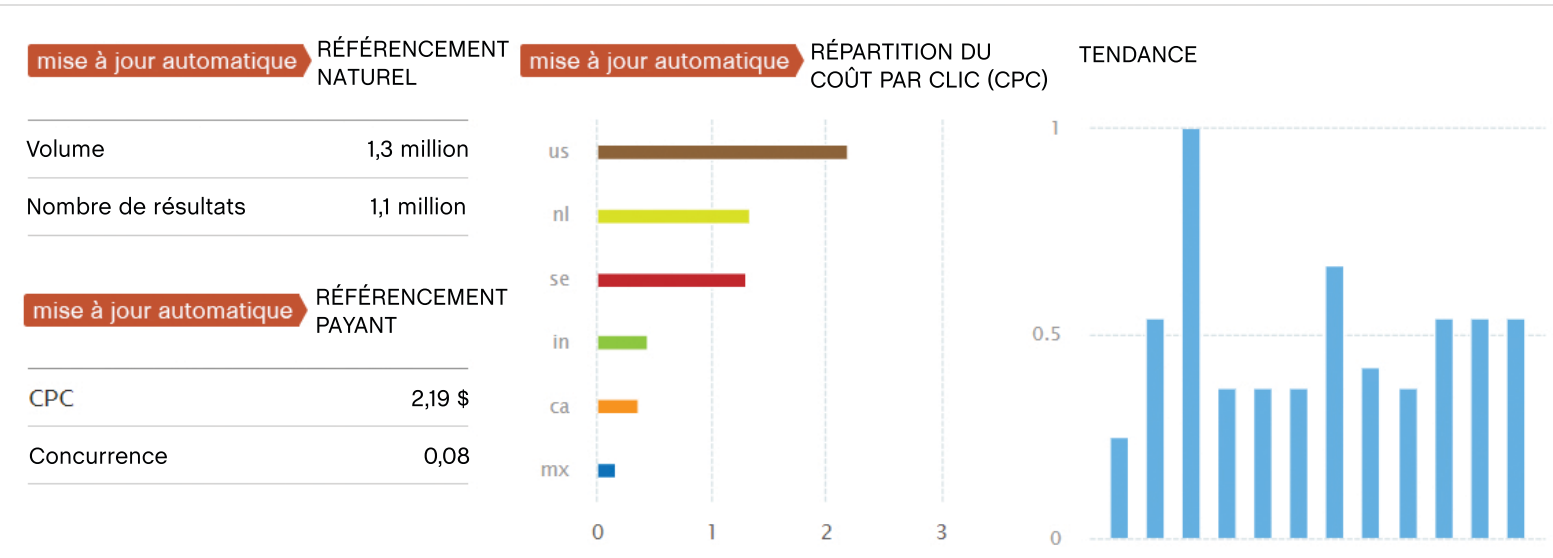

Allez-y, faites une recherche avec ce mot-clé. Avez-vous remarqué que deux cabinets d'optométrie figurent parmi les résultats? Ce pourrait être le vôtre. Après tout, y a-t-il une personne mieux placée qu'un optométriste pour répondre à cette question? 
Que se passe-t-il lorsque vous écrivez un article et que celui-ci est cité, référencé et partagé? Le graphique tend à ressembler à ce qui suit :

\section{Navigateur}

\section{Synthèse Utilisation du site Ensemble d'objectifs 1 Commerce électronique}

Utilisateur par rapport à Choisissez une mesure

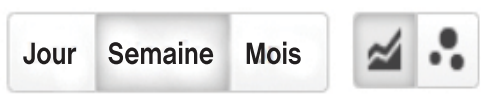

\section{Utilisateurs}

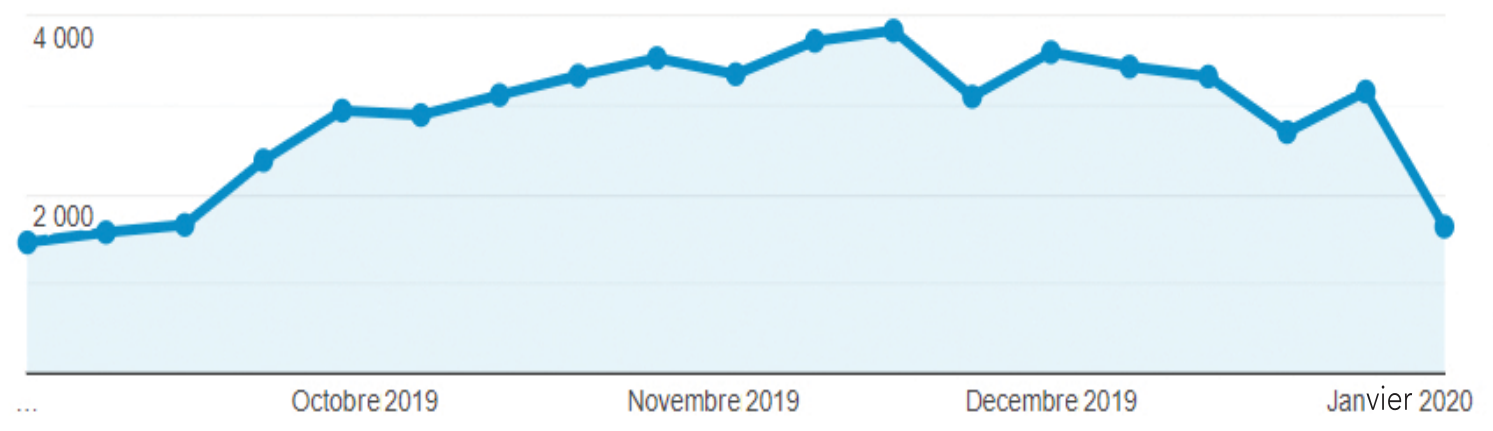

Cette capture d'écran, tirée de Google Analytics, montre le trafic hebdomadaire sur le blogue d'un cabinet d'optométrie du Maryland après la publication d'un article à la fin du mois de septembre.

Note : Cet article a été rédigé au début du mois de janvier. C'est pourquoi les chiffres de janvier semblent si bas en comparaison avec ceux des autres mois.

\section{Ils vous permettent de générer des clients potentiels... beaucoup de clients.}

Il s'agit de l'avantage le plus concret (et le plus important). Jetez un coup d'œil au graphique ci-dessus tiré de Google Analytics : même si seulement 0,05\% du nouveau trafic permettait d'aller chercher un nouveau client ou de cibler un client potentiel pour l'entreprise présentée plus haut, celle-ci aurait généré jusqu’à 20 nouvelles pistes de clients potentiels par mois à partir d'un seul article de blogue.

Quelle en serait l'incidence pour votre entreprise?

\section{CONSACREZ DU TEMPS À VOTRE BLOGUE ET RÉCOLTEZ LES FRUITS DE VOTRE LABEUR}

La tenue d'un blogue est une méthode simple qui nécessite une courte période d'apprentissage. Consacrez du temps à la création de contenu de qualité riche en détail et partagez-le ensuite sur vos réseaux sociaux. Avec de la persévérance, vous obtiendrez un meilleur référencement et verrez de nouveaux clients franchir votre porte. Voilà pourquoi vous devriez commencer un blogue. $\bullet$ 